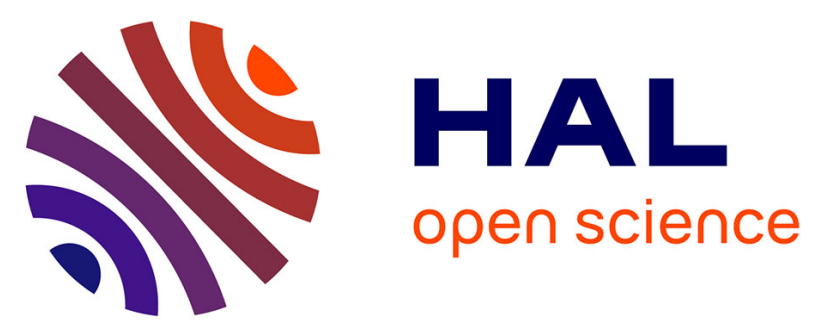

\title{
Multiple echoes due to distant dipolar fields in NMR of hyperpolarized noble gas solutions
}

Steven W. Morgan, Emmanuel Baudin, Gaspard Huber, Patrick Berthault, Geneviève Tastevin, Maurice Goldman, Pierre-Jean Nacher, Hervé Desvaux

\section{- To cite this version:}

Steven W. Morgan, Emmanuel Baudin, Gaspard Huber, Patrick Berthault, Geneviève Tastevin, et al.. Multiple echoes due to distant dipolar fields in NMR of hyperpolarized noble gas solutions. The European Physical Journal D: Atomic, molecular, optical and plasma physics, 2013, pp.67:29. 10.1140/epjd/e2012-30470-0 . hal-00762884v2

\section{HAL Id: hal-00762884 \\ https://hal.science/hal-00762884v2}

Submitted on 20 Jan 2013

HAL is a multi-disciplinary open access archive for the deposit and dissemination of scientific research documents, whether they are published or not. The documents may come from teaching and research institutions in France or abroad, or from public or private research centers.
L'archive ouverte pluridisciplinaire HAL, est destinée au dépôt et à la diffusion de documents scientifiques de niveau recherche, publiés ou non, émanant des établissements d'enseignement et de recherche français ou étrangers, des laboratoires publics ou privés. 


\title{
Multiple echoes due to distant dipolar fields in NMR of hyperpolarized noble gas solutions
}

\author{
Steven W. Morgan ${ }^{1}$, Emmanuel Baudin ${ }^{2}$, Gaspard Huber ${ }^{1}$, Patrick Berthault ${ }^{1}$, Geneviève \\ Tastevin $^{2}$, Maurice Goldman ${ }^{3}$, Pierre-Jean Nacher ${ }^{2}$, and Hervé Desvaux*1 \\ ${ }^{1}$ Laboratoire Structure et Dynamique par Résonance Magnétique, IRAMIS, CEA, CNRS, UMR 3299 SIS2M, CEA/Saclay, \\ 91191 Gif-sur-Yvette, France \\ ${ }^{2}$ Laboratoire Kastler Brossel, ENS; CNRS; UPMC; 24 Rue Lhomond, F75005 Paris, France \\ ${ }^{3} 6$ Résidence de Villebon, 91140 Villebon sur Yvette, France
}

\begin{abstract}
We report on multiple echo measurements in hyperpolarized liquids using optically pumped spin- $1 / 2$ noble gas atoms: either ${ }^{129} \mathrm{Xe}$ dissolved in cyclohexane or ${ }^{3} \mathrm{He}$ dissolved in superfluid ${ }^{4} \mathrm{He}$. An NMR pulse sequence $90^{\circ}-\tau-90^{\circ}$ (with slice-selective flipping pulses for ${ }^{129} \mathrm{Xe}$ experiments) was used and long echo trains have been observed in the presence of applied gradients due to average dipolar fields typically one order of magnitude larger than those of bulk water in high magnetic fields. We show that a mean field description is valid for explaining the multiple echoes observed in these liquids, even for spin temperatures as low as $10 \mathrm{mK}$ for ${ }^{129} \mathrm{Xe}$ or $10 \mu \mathrm{K}$ for ${ }^{3} \mathrm{He}$, and the echoes originate from the distant dipolar fields within the samples. Numerical lattice simulations have been used to assess the effects of slice selection and of finite sample size in addition to those of atomic diffusion. They account for the observed echo widths and amplitudes much better than previously published models which disregard finite size effects that appear to be of key importance. This opens the way to using multiple echoes resulting from distant dipolar fields for the determination of the absolute magnetization in hyperpolarized liquids without signal calibration.
\end{abstract}

\section{Introduction}

Since the observation by Deville et al. [1] of multiple echoes in solid ${ }^{3} \mathrm{He}$, numerous experiments exploring the effects of distant dipolar fields in liquids have been reported, in particular using thermally polarized water or liquid ${ }^{3} \mathrm{He}$ in high magnetic fields [2-5]. There are two ways to describe the observed phenomena that have been shown to be equivalent [6]. On one hand, a mean field theory has been used [1,7-10]: the strong magnetization of the sample induces a dipolar field that is experienced by the magnetic moments. This leads to non-linear terms in the Bloch equations describing the time evolution of the magnetization and explains the observation of multiple echoes. The mean-field approach is particularly useful for performing numerical simulations $[9,11]$. On the other hand, following the observation of "unexpected" echoes in the Correlated 2D spectroscopy Revamped by Asymmetric Z-gradient Echo Detection (CRAZED) experiments [12], a density matrix-based approach was proposed [13], relying on a claimed breakdown of the high temperature approximation $[12,13]$, with the introduction of intermolecular Multiple-Quantum Coherences (iMQC) [14]. The main advantage of this framework resides in its ability to more easily make analytical predictions. In particular, it has allowed the derivation of pulse sequences designed for real applications, for instance for performing
NMR or MRI in inhomogeneous magnetic fields [15-17] or media [18-22].

Beyond the usual range of average dipolar fields produced by bulk water, the domain of large dipolar fields in solutions has also been investigated using numerical simulations [23,24] and highly polarized ${ }^{3} \mathrm{He}$ and ${ }^{129} \mathrm{Xe}[25-28]$. The larger range of accessible densities and nuclear polarizations using these hyperpolarized nuclei has revealed the potential existence of collective behaviors observed as spectral clustering [29], chaotic time evolution leading to a rapid crunch of the magnetization [23,30-33] which can nevertheless be delayed by repeated magic sandwich sequences [34], and the observation of multiple maser emissions $[35,36]$. More recently two groups $[37,38]$ have reported the use of the generic CRAZED approach for observing i-MQC $[12,39]$ in ${ }^{13} \mathrm{C}$ or ${ }^{1} \mathrm{H}$ samples whose magnetization was previously enhanced by Dynamic Nuclear Polarization (DNP). We chose to perform multiple echo experiments in laser-polarized ${ }^{3} \mathrm{He}$ and ${ }^{129} \mathrm{Xe}$ samples for several reasons:

- higher polarizations $(>30 \%)$ than that obtained by brute force or by ex situ DNP can routinely be produced. This allows the exploration of very low spin temperatures and also large average dipolar fields when compared to that produced by bulk water in high magnetic field;

- these systems exhibit long longitudinal and trans-

\footnotetext{
*herve.desvaux@cea.fr, corresponding author.
} 
verse relaxation times (minutes). This permits several experiments with one polarization batch as well as more in-depth studies;

- the absence of scalar couplings allows direct comparisons between the experimental and numerical results.

In the present paper, we show that even with a high transient polarization, i.e. a low spin temperature (down to $10 \mathrm{mK}$ for ${ }^{129} \mathrm{Xe}$ at $11.7 \mathrm{~T}$ or $10 \mu \mathrm{K}$ for ${ }^{3} \mathrm{He}$ at $2.3 \mathrm{mT}$ ), the descriptions of the spin dynamics in terms of the density matrix or average dipolar field are equivalent. This proves that unexpected effects such as the predicted increased spectral interferences in highly polarized liquids [40] or the observed spectral narrowing in solids [41] are still negligible in the range of polarization we used. To investigate this regime in liquid systems with low transient spin temperatures, we have chosen to use a pulse sequence composed of two slice-selective $90^{\circ}$ pulses with a pulsed encoding gradient applied between them and an acquisition in the presence of a continuous gradient to observe many echoes (see Fig. 1). We used slice selection primarily to allow multiple experiments with each sample. The detection of several echoes allows us to compare experimental measurements to analytical and numerical predictions in a self-consistent way. The final aim of this work is to establish a method for absolute magnetization determination without resorting to external references, the latter being always loosely defined for transiently polarized spin systems. Several steps forward are reported in the present paper and in particular we demonstrate that finite size effects, which have been fully disregarded for several decades until very recently [42], appear of key importance. Finally the large range of dipolar fields accessible with our experiments has allowed us to observe strong distortions of the echoes for large dipolar fields (significant relative to the applied gradients).

\section{Theory}

\subsection{Spin temperature for an ex situ hy- perpolarized species}

A widespread approach for describing the observation of multiple dipolar echoes in liquids due to distant dipolar fields is to consider a spin system composed of all spins of the sample and the full Hamiltonian (including Zeeman terms and intermolecular dipolar interactions). Then it is usually argued that "there is a breakdown of the hightemperature approximation" [12,13], so that the full expansion of the density matrix has to be considered:

$\sigma=1+\alpha^{(1)} \sum_{n} I_{z}^{n}+\alpha^{(2)} \sum_{n<m} I_{z}^{n} I_{z}^{m}+\alpha^{(3)} \sum_{n<m<l} I_{z}^{n} I_{z}^{m} I_{z}^{l}+\cdots$

where at thermal equilibrium the different coefficients $\alpha^{(k)}$ are:

$$
\alpha^{(k)}=2^{k} \tanh ^{k}\left(\beta_{L} \gamma B_{0} / 2\right),
$$

with $\beta_{L}$ the inverse spin temperature of the lattice, $\gamma$ the gyromagnetic ratio, and $B_{0}$ the static magnetic field. The usual high temperature approximation consists in restricting the development of Eq. 1 to the first two terms and to linearize $\alpha^{(1)} \simeq \beta_{L} \gamma B_{0}$. Using the whole development of the density matrix (Eq. 1), one can predict the observation of multiple echoes following a $90^{\circ}-\tau-90^{\circ}$ excitation [12]. Essentially the $n^{\text {th }}$ echo results from the intermolecular $n$ spin-order coherences excited by the first $90^{\circ}$ hard pulse and that precess during $\tau$ as $n$ quantum coherences. This justifies the term i-MQC. The amplitude of this echo is directly related to the density matrix expansion (Eq. 1) and thus to the coefficient $\alpha^{(n)}$.

If we consider now an ex situ hyperpolarized spin system, before any rf pulse the density matrix can still be written as a series expansion of $I_{z}^{n} I_{z}^{m} I_{z}^{l} \ldots$, that is, according to Eq. 1. Treating the problem in this usual way immediately raises intriguing questions: What are the values of coefficients $\alpha^{(k)}$ in Eq. 1? Can they still be computed through Eq. 2 after the replacement of $\beta_{L}$ by the inverse spin temperature? Or in other words is the spin temperature still a well-defined quantity?

For any ex situ hyperpolarization mechanism, at the instant corresponding to the end of the polarization step the populations of the different states of the whole system are distributed according to a single spin temperature. After that moment, the system is out-of-equilibrium and is going to evolve towards thermal equilibrium. No mechanism in liquids can warrant the persistence of a spin temperature during this period since the intermolecular dipolar interactions are too weak to ensure efficient energy exchange. However, longitudinal relaxation is going to alter the distribution of spin-state populations since the relaxation rate of the single-spin order is different from the relaxation rates of intra- and inter-molecular multi-spin coherences. Thus addressed in such a way, the natural response corresponds to at least a large doubt on the existence of a spin temperature for an ex situ hyperpolarized spin system.

This difficulty is illustrative of the misleading message associated with a claim of "the breakdown of the hightemperature approximation." The main feature of a liquid is the absence of long distance correlations. ${ }^{1}$ Thus the density matrix of the whole spin system can always be written as a product of single molecule density matrices $\sigma_{m}$ :

$$
\sigma=\prod_{m} \sigma_{m}
$$

The restriction to single spin $1 / 2(I)$ atoms or molecules (such as ${ }^{129} \mathrm{Xe}$ or ${ }^{3} \mathrm{He}$ ) allows one to express the diagonal part of any individual $(2 \times 2)$ density matrix $\sigma_{m}$ as a function of the initial polarization $P_{0}=-\tanh \left(\beta_{I} \gamma B_{0} / 2\right)$ with $\beta_{I}$ the inverse nuclear spin temperature:

$$
\sigma_{m}=1+2 P_{0} I_{z}^{m} .
$$

\footnotetext{
${ }^{1}$ Local correlations exist in a liquid but Brownian motion rapidly averages out short-range intermolecular dipolar interactions and they can consequently be disregarded.
} 
Longitudinal relaxation is going to alter the polarization:

$$
P(t)=P_{0} \exp \left(-t / T_{1}\right)
$$

where $T_{1}$ is the longitudinal self-relaxation time of the spin species $I$ and where we have neglected the thermal equilibrium polarization relative to the transient value. The whole density matrix $\sigma$ at arbitrary time is given by Eq. 3 and the coefficients $\alpha^{(k)}$ are obtained by power expansion. With the key assumptions of a unique spin species and homogeneous evolution (single $T_{1}$ for all spins of the sample), the coefficients $\alpha^{(k)}$ are distributed according to a single-spin temperature.

The assumption of homogeneous evolution is intrinsically correlated to the transport properties of the studied system, since diffusion and convection will always tend to erease inhomogeneities at least at small enough scales. If non-uniform conditions remain over large distances, it remains possible to define position-dependent spin temperatures. The assumption of a unique spin species is more delicate. In fact, the existence of a spin temperature is not valid for transiently polarized multi-spin molecules such as those obtained by DNP $[37,38,43,44]$. Indeed, correlations between spins within the same molecule are preserved and the different intra-molecular spin-order coherences have different relaxation rates; for instance, due to the mutual dipolar interaction, the longitudinal relaxation rate of a two-spin order $2 I_{z}^{k} I_{z}^{l}$ is never equal to the sum of the one-spin relaxation rate of each spin (see for instance Ref. [45] for rate expressions). Thus effects due to distant dipolar fields and local scalar couplings are going to superimpose leading to multiplet intensities and splittings of coupled spins whose values differ from the dilute and thermal equilibrium ones [46].

This discussion illustrates that in the density matrix approach the key element for explaining experiments dealing with distant dipolar fields actually resides in the breakdown of the average molecule approximation. Indeed the usual procedure for solution-state NMR resides in considering for the Hamiltonian and the density matrix a spin system restricted to that of one single average solute molecule. Now when distant dipolar fields are studied, i.e. intermolecular interactions are considered, the description should be based on the tensor products of all individual density matrices (Eq. 3). Each one can be in the low spin temperature limit (for hyperpolarized spin systems) or in the high spin temperature approximation $\left(\beta_{L} \gamma B_{0} \ll 1\right.$, for thermally polarized samples).

\subsection{Multiple echoes due to distant dipo- lar fields}

The restriction to a single-spin molecule without correlations between molecules has as a consequence the equivalence between a one-spin density matrix description and a classical local magnetization description. Considering a thermally polarized single spin system, Jeener has shown the equivalence between the density matrix description and the mean field approach for experiments dealing with distant dipolar fields [6]. Obviously since we have shown that a spin temperature can be rigorously defined for a transiently polarized single spin system and since in our considered spin system the longitudinal self-relaxation times (several minutes) are much longer than the pulse sequence duration, the demonstration of equivalence done by Jeener can straightforwardly be extended to this case by introducing the transient spin temperature in place of the thermal equilibrium value. Within this framework, one can consequently use either the density matrix approach or the mean field approach.

In the rest of the present paper, we follow the initial description by Deville et al. [1] based on the classical mean field approach. In their experiments, multiple echoes were observed after two hard $90^{\circ}$ pulses separated by a delay $\tau$ in the presence of a continuous gradient of amplitude $G$ such that $G l$ (where $l$ is the relevant length of the sample) significantly exceeds the dipolar field associated with the magnetization (Sec. 4.3 discusses results obtained at higher magnetizations). When one disregards effects of diffusion and relaxation and in the absence of rf irradiation, the equation of time evolution of the local magnetization $\mathbf{M}$ is:

$$
\frac{\partial}{\partial t} \mathbf{M}=\gamma \mathbf{M} \times\left[\left(B_{0}+G z\right) \hat{\mathbf{z}}+\mathbf{B}_{\operatorname{dip}}(\mathbf{r}, t)\right]
$$

where the first term corresponds to the Zeeman contributions due to the collinear static magnetic field and gradient (aligned with the sample axis direction $\hat{\mathbf{z}}$ ), and the second to the time- and space-dependent dipolar field $\mathbf{B}_{\text {dip }}(\mathbf{r}, t)$ produced by the magnetization. During the acquisition, the transverse magnetization expressed in the rotating frame can usually be approximated for wellresolved echoes (with duration much shorter than the inter-echo time $\tau$ ) by [9]:

$$
M_{+}\left(t_{2}\right)=-M_{0} \int_{V} d z \sum_{n=-\infty}^{\infty} \imath^{-n} e^{-\imath \gamma z\left(G t_{2}-n G \tau\right)} \frac{n J_{n}\left(\omega_{\operatorname{dip}} t_{2}\right)}{\omega_{\text {dip }} t_{2}}
$$

where the integral is performed on the sample volume $V, J_{n}$ is the $n^{\text {th }}$ Bessel function of the first kind, and $\omega_{\text {dip }}=2 \pi f_{\text {dip }}=\mu_{0} \gamma M_{0}$ is the dipolar angular frequency associated with the uniform initial magnetization $M_{0}$. In this formulation, there is no specific condition on the polarization of the nuclear spins. The analytical solution (Eq. 7) has been established for homogeneous systems in which relaxation, diffusion, and edge effects can be neglected $[1,9]$.

In our experiments on polarized ${ }^{129} \mathrm{Xe}$, we have used a pulse sequence (Fig. 1) that differs from that of Deville et al. [1] in two respects. Slice-selective rf pulses have been used so as to excite only a small fraction of the sample and a pulsed encoding gradient of amplitude $G_{\mathrm{e}}$ and duration $\tau_{\mathrm{e}}$, applied just before the second rf pulse, replaced the constant encoding gradient so as to reduce the initial diffusion-induced signal attenuation. Since the am- 
plitudes of the gradients satisfy $G \tau=G_{\mathrm{e}} \tau_{\mathrm{e}}$, a series of echoes at times $t_{2}=n \tau$ is also expected.

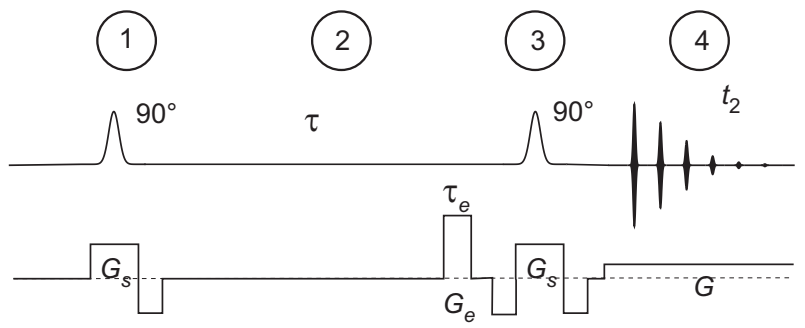

Figure 1: Pulse sequence used to investigate multiple echoes with polarized ${ }^{129} \mathrm{Xe}$. In the first (1) and third (3) periods the magnetization is slice selectively excited by $90^{\circ}$ Gaussian pulses combined with gradient $G_{\mathrm{S}}$. It evolves during the delay $\tau$ and the transverse component is encoded by the gradient $G_{e}$ with a duration $\tau_{e}$ that satisfies $G_{e} \tau_{e}=G \tau$. Echoes are detected during the acquisition period (4), during which a constant gradient $G$ is applied leading to the appearance of echoes separated by the delay $\tau$. For the ${ }^{3} \mathrm{He}$ experiments a constant gradient $G$ is applied throughout the experiment and no slice selection is applied for excitation which only consists of square rf pulses. All gradients are applied along the longitudinal axis of the sample ( $\hat{\mathbf{z}}$ gradient).

In our experiments, as well as in previous studies of multiple echoes due to distant dipolar fields [1-5], the two rf pulses have the same phase. However, the encoding gradient applied between the rf pulses locally shifts the phase of the precessing magnetization and the relative phase of the rf pulses plays no role for $\gamma G_{e} \tau_{e} \gg 1$. This contrasts with solid-state NMR experiments performed without applied gradients for which the relative phase of the rf pulses is important, with for instance a solid-echo following a $90_{x}^{\circ}-90_{y}^{\circ}$ but not a $90_{x}^{\circ}-90_{x}^{\circ}$ pulse pair [47].

\section{Experimental methods}

\subsection{NMR experiments}

Most experiments have been performed with hyperpolarized ${ }^{129} \mathrm{Xe}$ produced by spin-exchange optical pumping of rubidium using an experimental apparatus that works in batch mode $[48,49]$. Up to four batches of $86 \%$ enriched ${ }^{129} \mathrm{Xe}$, were accumulated before transferring by cryo-condensation into a previously degassed NMR tube. The tube, of $3 \mathrm{~mm}$ outer diameter and $1.6 \mathrm{~mm}$ inner diameter, contained $100 \mu \mathrm{L}$ of deuterated cyclohexane. The diffusion coefficient of xenon under these conditions is $2.1 \cdot 10^{-9} \mathrm{~m}^{2} / \mathrm{s}$. The initial polarization of the dissolved xenon was up to $30 \%$. Polarization measurements were made by comparing the hyperpolarized xenon NMR signal after a small excitation pulse to the overnight average thermal equilibrium signal obtained after the same excitation pulse. The xenon concentration was determined after the experiment by weighing the NMR tube before and after degassing the xenon and using the reported xenon solubility in cyclohexane [50]. The values of the extracted dipolar frequencies $f_{\text {dip }}$ were checked to be consistent with those directly determined from ${ }^{1} \mathrm{H}$ frequency shifts measured in interleaved experiments [51]. $f_{\text {dip }}$ values ranged up to $9 \mathrm{~Hz}$, which corresponded to dipolar fields (760 nT) more than 15 times that of thermally polarized bulk water at room temperature and $11.7 \mathrm{~T}(48 \mathrm{nT})$.

All xenon experiments were run on a Bruker Avance II $500 \mathrm{MHz}$ spectrometer. A 5-mm inverse triple resonance probe head was used, where the broadband channel used for xenon excitation and detection corresponded to the outer coil. This choice reduced contributions due to radiation damping, which were already limited by the low gyromagnetic ratio of ${ }^{129} \mathrm{Xe}$, since radiation damping rate scales as $\gamma^{3}$ at thermal polarization. The maximum radiation damping contribution for such an experimental configuration was estimated from previous measurements [36], to be smaller than $1 \mathrm{~s}^{-1}$. The circuit for the xenon channel was tuned for optimal reception (perfect agreement between the resonance frequency of the electronic reception circuit and the Larmor frequency) [52]. The magnetic field was locked to the ${ }^{2} \mathrm{H}$ signal of the solvent.

We used the pulse sequence of Fig. 1 to probe multiple echoes due to distant dipolar fields. The selected slice thickness determined by the gradient strength $G_{\mathrm{S}}$ and the duration of the soft Gaussian pulse $(196 \mu \mathrm{s}$ FWHM, $500 \mu \mathrm{s}$ full duration) was typically $1.8 \mathrm{~mm}$ FWHM for a gradient $G_{\mathrm{S}}=200 \mathrm{mT} / \mathrm{m}$. A typical run of experiments consisted of dissolving the polarized xenon and performing a series of signal acquisitions. Xenon polarization was refreshed between each measurement using the reservoir of gaseous xenon by vigorously shaking the NMR tube, ensuring homogeneous magnetization. Each measurement in the series was usually made by acquiring multiple echoes from three well-separated slices in a $40 \mathrm{~mm}$ long sample. The inter-echo time $\tau$ was varied between 45 and $120 \mathrm{~ms}$ and the gradient $G$ was typically $4 \mathrm{mT} / \mathrm{m}$.

We performed similar NMR experiments using liquid ${ }^{3} \mathrm{He}-{ }^{4} \mathrm{He}$ mixtures. ${ }^{3} \mathrm{He}$ gas was polarized using metastability-exchange optical pumping and subsequently dissolved in liquid ${ }^{4} \mathrm{He}$ at $T=1.3 \mathrm{~K}$, with initial polarization of up to $30 \%$ and concentrations such that $f_{\text {dip }}$ ranged up to $11 \mathrm{~Hz}[28,34]$. The diffusion coefficient of ${ }^{3} \mathrm{He}$ in these conditions was on the order of $10^{-7} \mathrm{~m}^{2} / \mathrm{s}$. No slice selection was used and a constant gradient of $1.3 \mathrm{mT} / \mathrm{m}$ was applied (eddy currents prevented fast gradient switching in these low temperature experiments). NMR was performed at $74 \mathrm{kHz}(2.3 \mathrm{mT})$, active feedback was used to reduce the effects of radiation damping [53], and the inter-echo times were less than $50 \mathrm{~ms}$.

Finally, the consistency of the experimental results was checked using bulk water at $11.7 \mathrm{~T}$, the same pulse sequence as for hyperpolarized xenon, and gradient values scaled down by the ratio $\gamma_{\mathrm{Xe}} / \gamma_{\mathrm{H}}$. 


\section{$3.2 \quad$ Numerical simulations}

Numerical simulations were based on an exact calculation of the time evolution of coupled magnetic moments on a cubic lattice. The simulations included the effects of diffusion, magnetic field gradients, dipolar interactions, and rf field including radiation damping. The software was initially developed by Jeener [23], using methods that were also used by other groups $[54,55]$. Computations were performed in the rf rotating frame using a standard secular approximation. The magnetic field induced at each site by the remainder of the sample was efficiently computed by toggling between real space and Fourier space using periodic boundary conditions $[1,9,54]$. The time evolution was computed by integrating the non-linear Bloch equations using a standard Runge-Kutta technique. Two kinds of simulations were performed: the case where edge effects were neglected was studied using a $1 \mathrm{D}$ unit cell to describe an infinite system with perfect translational invariance in the transverse directions $(\hat{\mathbf{x}}, \hat{\mathbf{y}})$. The case where finite size effects were taken into account was modeled using a 3 D unit cell of $158 \times 158 \times 600$ sites typically including $68 \times 68 \times 240$ magnetic moments. This corresponded to the aspect ratio of the xenon samples (although not to their cylindrical shape) but the computational load of such realistic simulations was quite heavy, of order a few days per second of physical time on a desktop computer.

\section{Results and discussion}

\subsection{Experimental observation of multiple echoes}

Figure 2 shows typical time domain signal amplitudes obtained using the pulse sequence of Fig. 1 and dissolved laser-polarized ${ }^{129} \mathrm{Xe}$. As predicted by Eq. 7, echoes appear at $t_{2}=n \tau$ where $n$ is an integer. For typical experimental conditions, well-resolved echoes are obtained (Fig. 2a). For a large magnetization and a short echo time, up to 14 echoes have been observed (Fig. 2b).

Except for the highest magnetizations, the echoes can be accurately fitted by Gaussian functions. This fitting procedure allows a measurement of the echo widths and a precise determination of the echo amplitudes. The echo widths are observed to increase with echo number (insets in Fig. 2). This increase is due to the use of a slice selection gradient and a soft pulse. It is also obtained in numerical lattice simulations when a slice is selected by a Gaussian pulse, whereas constant echo widths are obtained when magnetization is uniformly tipped with $90^{\circ}$ hard pulses.

A constant echo width is indeed observed in Fig. 3 where a series of echoes are detected on a mixture of ${ }^{3} \mathrm{He}-{ }^{4} \mathrm{He}$ after two non-selective $90^{\circ}$ hard pulses. For this near-hemispheric sample shape [34], a sinc-like echo shape is expected, with side lobes contributing to the observed broad baseline in the signal.
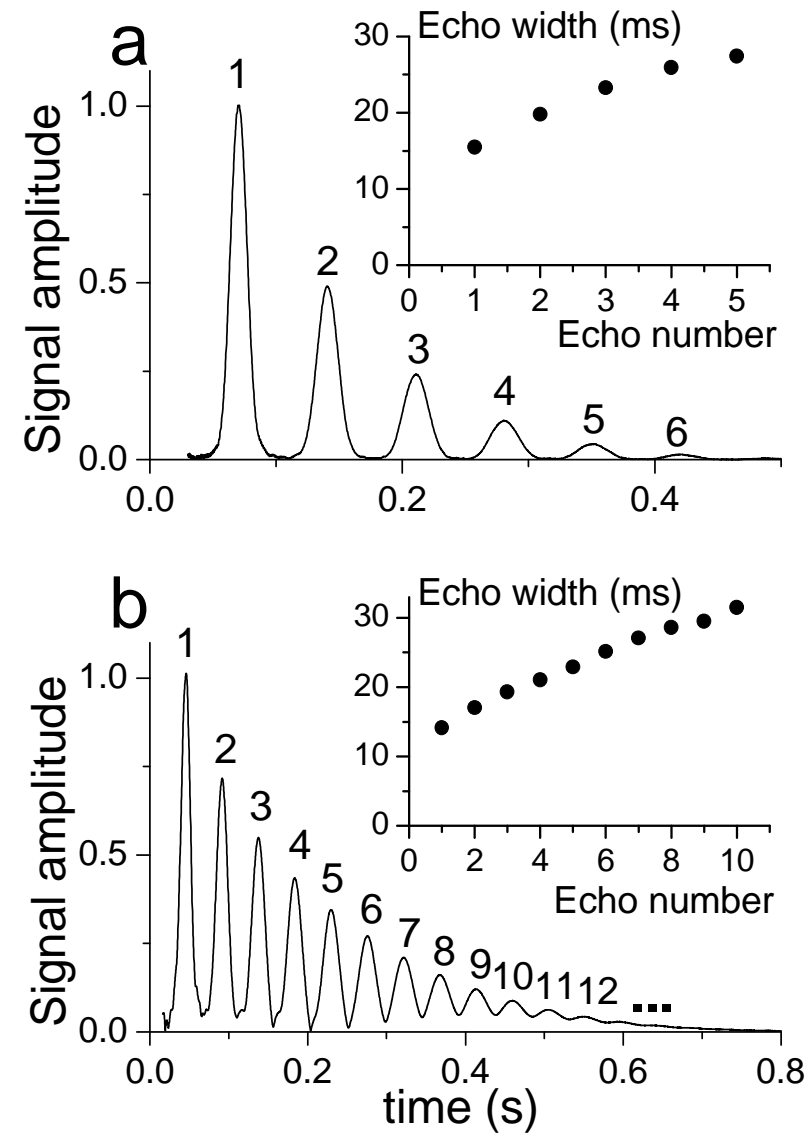

Figure 2: Time-domain variations of signal amplitudes obtained with the pulse sequence of Fig. 1 and dissolved laser-polarized xenon. a: For an inter-echo time $\tau=70 \mathrm{~ms}$ and $f_{\text {dip }}=3.1 \mathrm{~Hz}$, a series of Gaussian-shaped echoes is observed. b: A larger number of echoes is obtained with $\tau=45 \mathrm{~ms}$ and $f_{\text {dip }}=9 \mathrm{~Hz}$. The insets display the corresponding echo widths (FWHM).

One common feature of the ${ }^{3} \mathrm{He}$ and ${ }^{129} \mathrm{Xe}$ signals is the significant width of the echoes. For a constant read gradient $G$ value, the thinner is the slice selection, the broader is the echo width. This width results from the combined use of slice selection (for Xe samples) and detection gradients of moderate amplitudes. However, this choice of gradients also limits signal losses resulting from diffusion. Even though the widths of these echoes are smaller than those obtained by the multi-CRAZED approach where the magnetization is precessing in the absence of gradients $[12,39]$, their finite values prevent the use of very short inter-echo times $\tau$ unless either a larger sample slice thickness is selected or a stronger gradient $G$ is used.

\subsection{Comparison of analytical, numerical, and experimental echo amplitudes}

In the ideal situation considered for the derivation of Eq. 7 (infinite medium, no diffusion, no relaxation, uniform $90^{\circ}$ 


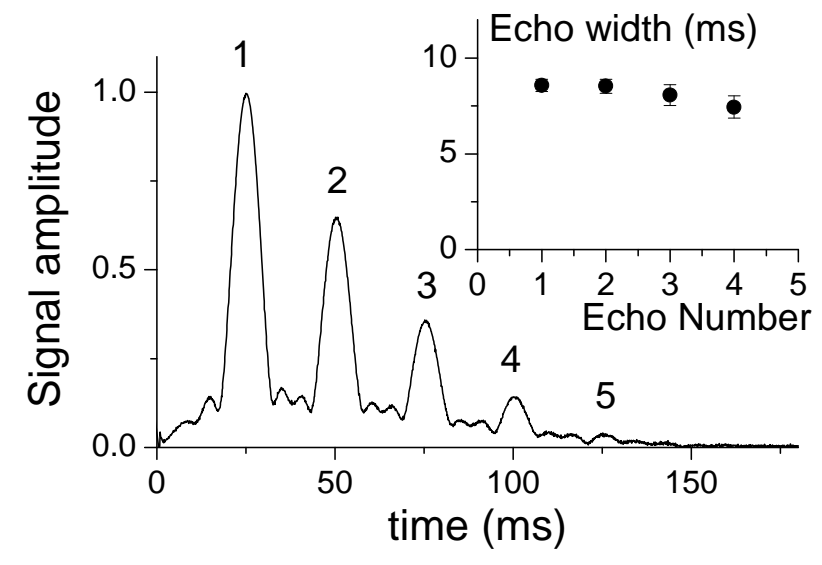

Figure 3: Time-domain variation of signal amplitude obtained with hyperpolarized ${ }^{3} \mathrm{He}$ and a constant gradient for $\tau=24.6 \mathrm{~ms}$ and $f_{\text {dip }}=5.5 \mathrm{~Hz}$. The diffusion coefficient of ${ }^{3} \mathrm{He}$ was 50 times larger than that of ${ }^{129} \mathrm{Xe}$, which impeded the observation of more numerous echoes. The inset displays the echo widths (FWHM) of the first echoes; they do not increase with echo number, in contrast to those of Fig. 2.

excitation pulse), the amplitude $S_{\text {id. }}^{(n)}$ of the signal detected for the $n^{\text {th }}$ echo is:

$$
S_{\text {id. }}^{(n)}=K M_{0} V \frac{2 n J_{n}\left(n \omega_{\mathrm{dip}} \tau\right)}{2 n \omega_{\mathrm{dip}} \tau}
$$

where $M_{0}$ is the magnetization at the time of the experiment and $K$ is the instrumental response factor. To avoid the dependence on uncertain parameters such as the thickness of the selected slice (affecting $V$ ) or the exact polarization at any particular time (affecting $M_{0}$ ) we resort to the reduced amplitudes $A_{\mathrm{id}}^{(n)}$, normalized to the amplitude of the first echo:

$$
A_{\mathrm{id} .}^{(n)}\left(\omega_{\mathrm{dip}} \tau\right)=\frac{S_{\mathrm{id} .}^{(n)}}{S_{\mathrm{id} .}^{(1)}}=\frac{J_{n}\left(n \omega_{\mathrm{dip}} \tau\right)}{J_{1}\left(\omega_{\mathrm{dip}} \tau\right)} .
$$

$A_{\text {id. }}^{(n)}$ only depends on the dimensionless quantity $\omega_{\operatorname{dip}} \tau$ where $\omega_{\text {dip }}$ scales with $M_{0}$. The reduced amplitude $A_{\text {id. }}^{(n)}$ monotonically increases with $\omega_{\operatorname{dip}} \tau$ (hence can be used to uniquely determine $\omega_{\text {dip }} \tau$ ) for values of this parameter up to 1.5. For larger values, the oscillatory nature of the Bessel functions may yield irregular echo patterns [9]. For the same reason of avoiding uncertain parameters, we also consider the reduced amplitudes $A^{(n)}=S^{(n)} / S^{(1)}$ for the echo amplitudes for the analytical, experimental, and numerical cases. ${ }^{2}$

Selected results of numerical lattice simulations are used to illustrate the effect of physical processes that are not considered in the simple description presented so far (Eqs. 6 and 7). Figures 4a and 4b display examples of echo

\footnotetext{
${ }^{2}$ Bowtell et al. [3] also used this ratio for monitoring the effects of diffusion.
}
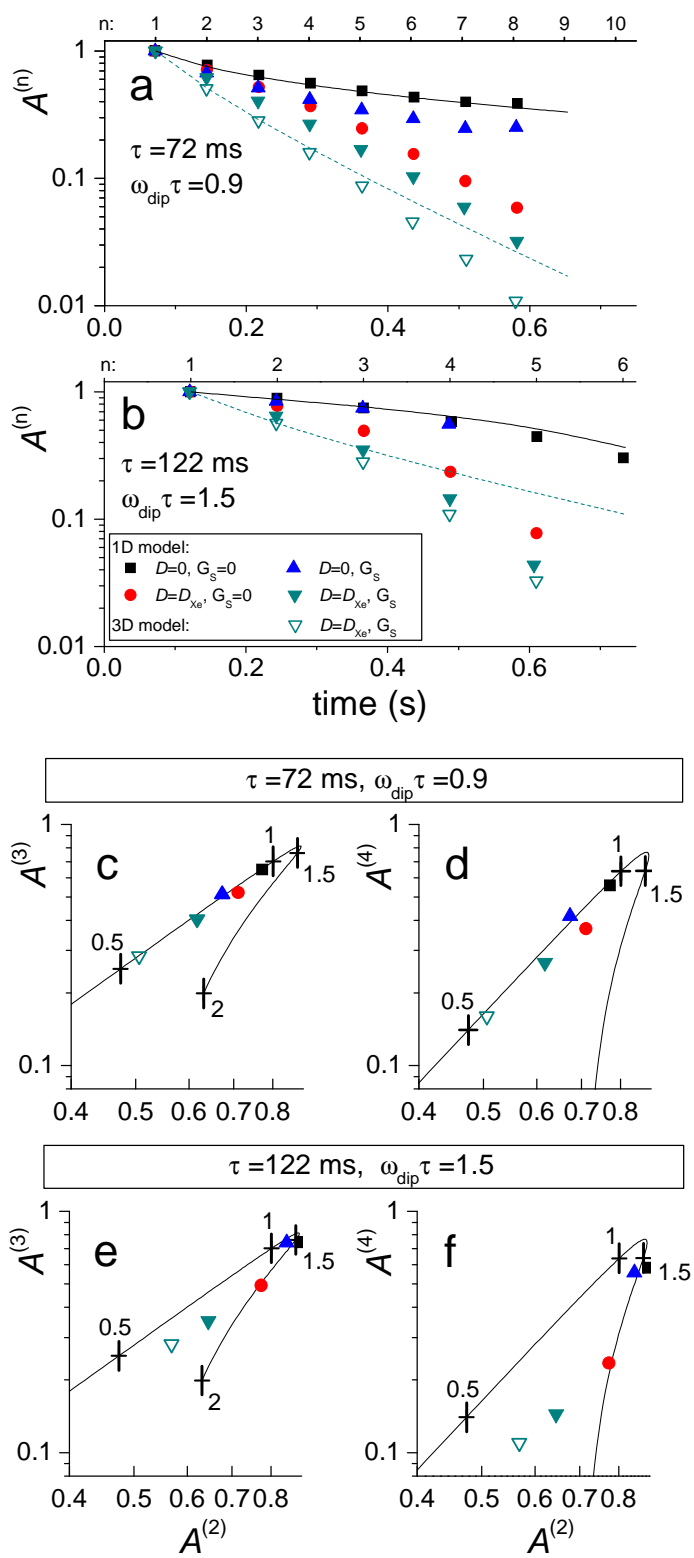

Figure 4: Results of lattice simulations with a $1 \mathrm{D}$ model (solid symbols) without or with slice selection $G_{\mathrm{S}}$ and diffusion attenuation $D_{\mathrm{Xe}}$ (see legend in plot b), or a full $3 \mathrm{D}$ model with $G_{\mathrm{S}}$ and $D_{\mathrm{Xe}}$ (open triangles). a, b: Reduced echo amplitudes for $f_{\text {dip }}=2 \mathrm{~Hz}$ and different inter echo times. The solid and dotted lines interpolate values of Eq. 9 at times $n \tau$ for the nominal $\omega_{\text {dip }} \tau$ value and an appropriately smaller $\omega_{\text {id. }} \tau$ value, respectively (see text). c,d (resp. e,f): Reduced amplitudes of the third and fourth echoes in a (resp. b), plotted as functions of the reduced amplitudes $A^{(2)}$. The solid lines are the ideal case amplitudes of Eq. 9, and the labeled + symbols correspond to the indicated values of $\omega_{\text {dip }} \tau$.

amplitudes obtained by computer simulations for a sample with infinite transverse size (1D model, solid symbols) and for a finite sample with dimensions corresponding to the xenon experiment (3D model, open triangles). The 1D 
simulations in the ideal situation $\left(D=0, G_{\mathrm{S}}=0\right.$, solid squares in Fig. 4) yield decaying echo amplitudes that are well described by the reduced ratios $A_{\text {id. }}^{(n)}\left(\omega_{\text {dip }} \tau\right)$ (Eq. 9, solid lines). Enhanced echo decays are obtained when slice selection, or diffusion, or both, are included. In contrast, radiation damping induces a negligible additional attenuation for rates corresponding to our experimental conditions.

The use of a Gaussian selective pulse yields weaker dipolar effects and smaller echo amplitudes. This is attributed to the weaker spatial modulation of the longitudinal magnetization induced at the boundaries of the excited slices. Diffusion-induced attenuation also decreases the amplitudes of the echoes and the relative effect of this attenuation is stronger at longer inter-echo time $\tau$ (Fig. 4b). When slice selection and diffusion are both included in the simulations, finite size effects further decrease the amplitudes of the echoes (full 3D model, open symbols in Fig. 4). The impact of this additional decrease is rather large (especially at short $\tau$, Fig. 4a), given that the sample diameter $(1.6 \mathrm{~mm}$, similar to the slice thickness) is larger than the modulation period of the magnetization $(0.3 \mathrm{~mm}$ for $\tau=70 \mathrm{~ms}, 0.18 \mathrm{~mm}$ for $\tau=120 \mathrm{~ms}$ ). A given echo train usually displays a non-exponential behavior, resembling a stretched exponential for negligible diffusion and $\omega_{\text {dip }} \tau<1$ (Fig. 4a) or a compressed exponential in opposite conditions (Fig. 4b).

For the same numerical results, the reduced amplitudes $A^{(3)}$ and $A^{(4)}$ of the third and fourth echoes are plotted as a function of $A^{(2)}$ in Figs. 4c to 4f. In this representation, the good agreement between simulations and reduced ratios of Bessel functions (Eq. 9, solid lines) that is obtained in the absence of diffusion and of a slice selection gradient is evidenced by the fact that corresponding data points $A^{(n)}$ (solid squares) coincide with $A_{\mathrm{id}}^{(n)}$ for the relevant value of $\omega_{\mathrm{dip}} \tau$. The enhanced decays due to diffusion, slice selection, or edge effects alter the correlation between the successive echo amplitudes in a way that, at fixed $\omega_{\text {dip }}$, depends on the inter-echo time $\tau$. At short echo time (Figs. 4c and 4d), all other simulations yield data points that approximately remain on the lines corresponding to the analytical solutions but lie at positions associated with smaller values of the parameter $\omega_{\text {dip }} \tau$. At long echo time (Figs. 4e and f) the echo amplitudes depart more and more from the ideal case expectations and the largest discrepancies are obtained for the simulations that include diffusion.

The dotted lines in Figs. 4a and 4b show the envelopes of the multiple echo trains obtained using Eq. 9 with effective values $\omega_{\text {id. }}$ of the dipolar frequency such that the ideal model exactly reproduces the results of $3 \mathrm{D}$ simulations for $A^{(2)}$ :

$$
A_{\text {id. }}^{(2)}\left(\omega_{\text {id. }} \tau\right)=A_{3 \mathrm{D}}^{(2)}\left(\omega_{\text {dip }} \tau\right)
$$

These effective values are significantly smaller than the actual input ones $\left(\omega_{\text {id. }} \tau=0.54\right.$ and 0.61 for $\omega_{\text {dip }} \tau=0.9$ and 1.5 , respectively), which is due to the enhanced second echo attenuation induced by the cumulative effects of slice selection, diffusion, and finite sample size. The ratios between the effective values $\omega_{\text {id. }}$ and the actual values $\omega_{\text {dip }}$ depend on the characteristics of both the physical system and the pulse sequence. Moreover, the amplitudes of the multiple echoes also strongly depend on these operating conditions. Therefore, for a given echo train, the comparison of amplitudes between the various echoes should provide a sensitive test to evaluate the relevance of theoretical or numerical models. This is the primary motivation for our experimental procedure, designed to allow the observation of a large number of echoes for various delay times and magnetization values, as well as for the choice of an intermediate echo time $(\tau=70 \mathrm{~ms})$ for performing extensive tests on the xenon samples.

Figure 5 shows a compilation of experimental results obtained with decay times $\tau$ ranging from 45 to $120 \mathrm{~ms}$ (symbols), for which the reduced echo amplitudes $A^{(3)}$, $A^{(4)}$, and $A^{(5)}$ plotted as a function of $A^{(2)}$ can be compared to the corresponding analytical solutions (Eq. 9, solid lines). For a wide range of echo attenuations, the data points lie close to the lines, with distances (below the
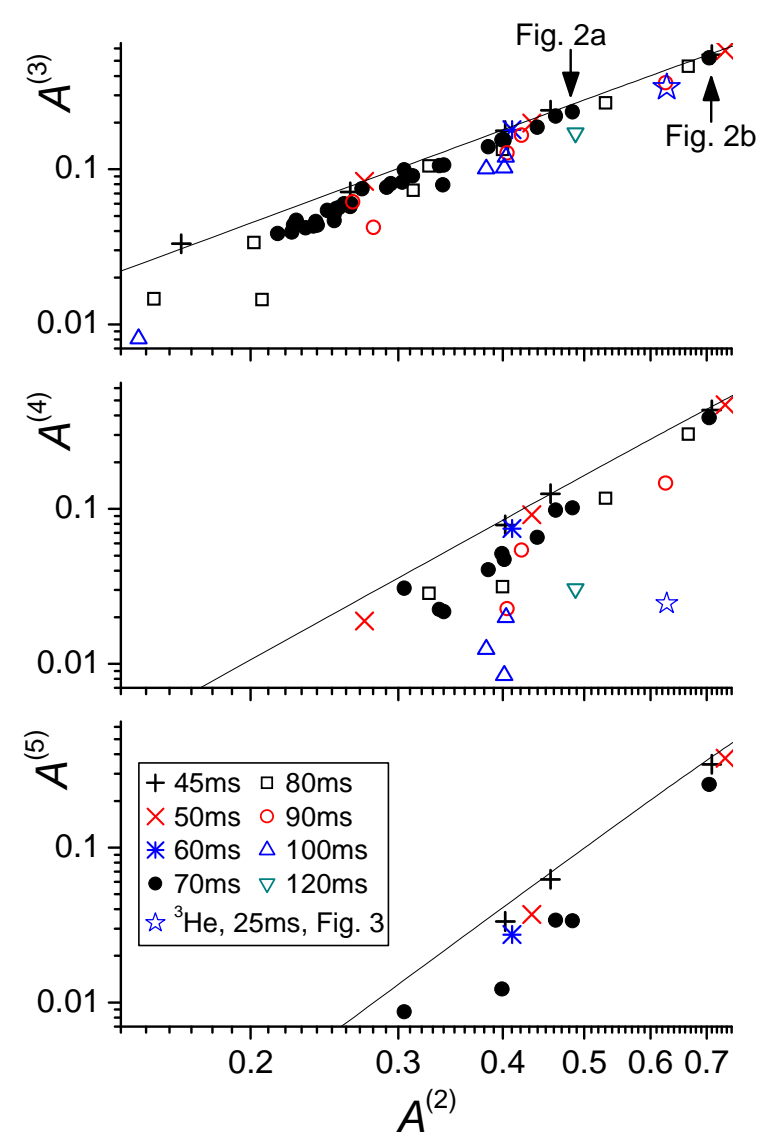

Figure 5: Experimental reduced echo amplitudes $A^{(3)}$ to $A^{(5)}$ (top to bottom) are plotted as functions of $A^{(2)}$ for various $\tau$ (symbols, see legend). The lines are the upper branches $\left(\omega_{\text {dip }} \tau<1.5\right)$ of the corresponding ideal echo amplitudes $A_{\text {id. }}^{(n)}$ (Eq. 9). The data from Fig. 2a $\left(\omega_{\text {dip }}^{\exp } \tau=1.4\right)$ and Fig. $2 \mathrm{~b}\left(\omega_{\text {dip }}^{\exp } \tau=2.5\right)$ are indicated by the labelled arrows. 
lines) that systematically increase with both $\tau$ and $n$. This is very similar to the results of the simulations (see Figs. 4c-4f). We can thus infer that slice selection, diffusion, and edge effects may all contribute to echo attenuation, with diffusion playing a leading role at long times (large $\tau$ and $n$ ) in accelerated echo decays. As was done for the comparison of results of 3D simulations to the ideal model (Eq. 10), we consider the effective dipolar frequency $\omega_{\text {id. }}$ for which the ideal model (Eq. 9) exactly reproduces the experimental reduced amplitudes $A^{(2)}$. For the recordings of Figs. 2a and 2b (data marked by arrows in the upper graph in Fig. 5, $\omega_{\text {dip }} \tau=1.4$ and 2.5, respectively), effective values $\omega_{\text {id. }} \tau=0.51$ and 0.81 are obtained. These large ratios between effective and externally determined dipolar frequencies are similar to those observed for 3D simulations, that provide a much better description of the observed attenuations than the ideal model. Indeed, the results displayed in Fig. 4 show that including the effects of diffusion in the model $[1,3]$ corrects for only part of the missing attenuation. Nevertheless, the ratios between the actual and effective dipolar frequency values in the experimental cases are still higher than expected from the full 3D lattice simulation (closer to 3 than 2). This quantitative difference remains to be clarified. A consequence of the strong reduction of the effective value of the dipolar frequency, is that we could not experimentally achieve effective values of $\omega_{\text {id. }} \tau$ larger than about 1.5 , for which the echo amplitudes are not monotonically decreasing. Similar behaviors and discrepancies were observed for bulk water data (not shown) and for the helium data. For the latter and for clarity, only the recording displayed in Fig. 3 was used here for comparison to the xenon data, open star in Fig. 5. In this case, diffusion attenuation is significant at shorter $\tau$ and current 3D simulations account fairly well for the measured ratio between the effective and experimental values $\left(\omega_{\text {id. }} \tau=0.7\right.$ and $\omega_{\text {dip }} \tau=0.85$, respectively).

Obtaining a more quantitative model of echo attenuations to infer the sample magnetization would fully establish the practical interest of the multiple echo technique. This ability to quantify $f_{\text {dip }}$ for an ex situ transiently polarized spin system in a one-scan experiment would represent a good alternative to other methods, such as:

monitoring the dipolar-induced shifts of the resonance frequencies $[51,56]$; this forbids the lock of the magnet and is consequently sensitive to perturbations (e.g., temperature or magnetic fluctuations). Therefore only a continuous frequency monitoring allows a reliable determination of $f_{\text {dip }}$;

using signal measurements after small excitation pulses, referenced to thermal equilibrium signals and concentration measurements; these two additional measurements are either relatively long if performed on the sample of interest or sensitive to systematic bias if external references are used.

Finally an important additional advantage of the multiple- echo approach would reside in the use of slice-selection since the determination of $f_{\text {dip }}$ would alter only a small part of the sample magnetization while the rest is preserved and can be used for other types of experiments.

\subsection{Echo distortion and instabilities}

Numerical lattice simulations allow the investigation of a range of $f_{\text {dip }}$ values beyond those where the simple analytical model is valid $[1,9]$. Figure 6 shows the evolution of computed multiple echoes for increasing dipolar fields, obtained using the 3D model for a finite sample with selective excitation and diffusion. The increase of $f_{\text {dip }}$ first leads to an increase in the number of echoes, as expected from the analytical solution of the ideal case. When $f_{\text {dip }}$ further increases, the shape of the echoes starts to be distorted, and the echoes eventually disappear. Since significantly larger values of average dipolar fields can be produced by resorting to hyperpolarized species, we experimentally observed signals showing such distortions, with the appearance of small lobes beside the echoes (inset of Fig. 6 for dissolved laser-polarized ${ }^{129} \mathrm{Xe}$ ). As was noticed in the previous section, there is a fair agreement between numerical simulations and experimental observations, however a $\sim 50 \%$ quantitative difference in the values of $f_{\text {dip }}$ still needs to be accounted for. Beside numerical restraints such as unsufficiently fine graining

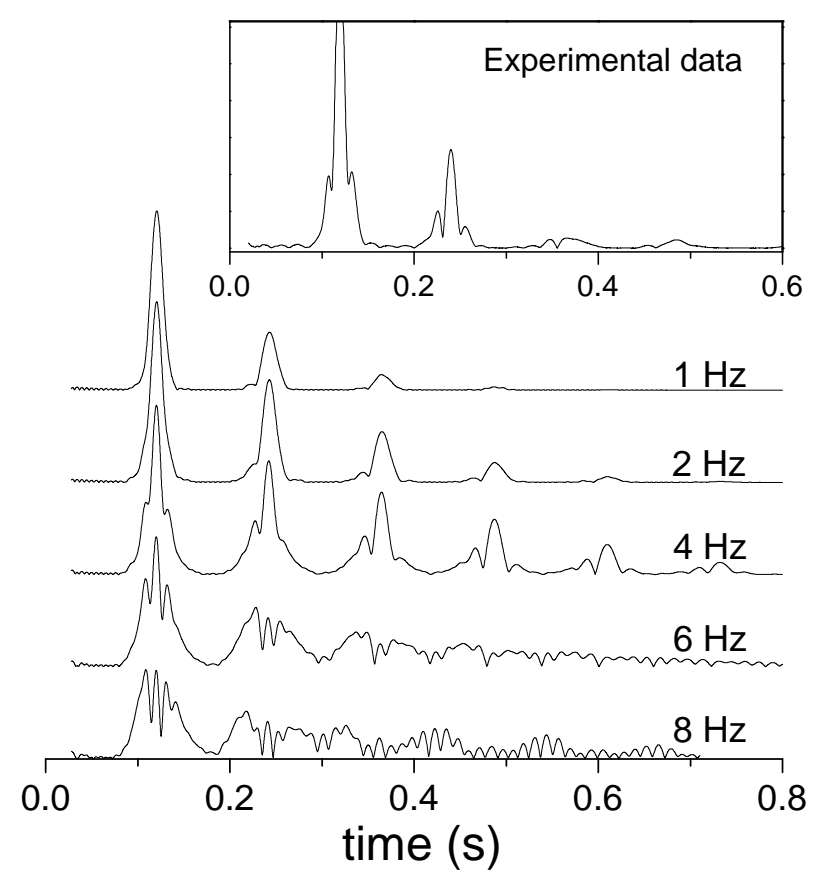

Figure 6: Main plot: numerical 3D lattice simulations of the variation of the echo pattern as a function of $f_{\text {dip }}$ for $\tau=120 \mathrm{~ms}$ (with slice-selection and taking into account xenon diffusion). Inset: experimental result with a dissolved laser-polarized xenon sample $\left(f_{\text {dip }}=9 \mathrm{~Hz}\right)$. The vertical scale is chosen so as to better visualize the altered echo shapes. 
which prevent carefully considering edge effects, this discrepancy may also result from the large sensitivity of the xenon chemical shifts to its surrounding which affects the dipolar flip-flop contributions to the time evolution of the magnetization [10].

The origin of the changes in the shape and number of observed echoes lies in the decreasing efficiency of the applied gradients to refocus the effects of the dipolar field $[1,9]$ since the condition $f_{\text {dip }} \ll \gamma G l / 2 \pi$ is no longer fulfilled (the gradient-induced frequency span in our xenon experiments is approximatively equal to $80 \mathrm{~Hz}$ ). For numerical simulations with larger gradients the distorted echoes indeed appear for larger $f_{\text {dip }}$ values. In the original paper on multiple echoes due to distant dipolar fields [1], Deville et al. show situations where the condition of strong enough gradients is not met. In their case, the echo amplitudes increased with time. Here we have used strong enough gradients to prevent the overlap of the echoes. However, the cumulative effect of precession instabilities (similar to those previously described numerically and observed experimentally $[23,26,28,31])$ gave rise to the echo distortions observed in this work and prevented the observation of a large number of echoes for large values of $f_{\text {dip }}$ and $\tau$.

\section{Conclusions}

In this article we have explored multiple spin echoes resulting from distant dipolar fields in solutions containing laser-polarized xenon-129 or helium-3. The use of these spin systems has allowed the investigation of a large range of conditions in terms of magnetization, spin temperature, diffusion constants, etc ., well beyond what can be explored using thermally polarized spin systems. We have first shown that the usual frameworks (mean field theory or an extended density matrix approach) can be used to describe the dynamics of these hyperpolarized spin systems since they fulfill the two key conditions: (i) protocols are used to ensure an homogeneous evolution of the magnetization described by a single longitudinal selfrelaxation time and (ii) the system only consists of singlespin molecules.

The method proposed in the present paper should make it possible to determine the absolute magnetization without any separate experiments, just by comparing the ratios of echo amplitudes. This would be especially useful since it would allow the determination of the sample magnetization using only a small slice of the sample while the rest of the sample's magnetization is preserved. Resorting to the processing method based on ratios of echo amplitudes allows its use for direct and quantitative comparisons with numerical predictions, and not only qualitative comparisons of behaviors. Actually, the meanfield computations are in fair agreement with our measurements, even for very low spin temperatures. A still unsolved quantitative discrepancy (of about a factor 1.5) is observed between the experimentally measured dipolar field values $f_{\text {dip }}$ (obtained from ${ }^{1} \mathrm{H}$ dipolar shift measurements or from polarization and concentration measurements) and those used in the numerical simulations for observing the same echo amplitudes. It might result in part from the numerical restraints that currently prevent the simulation of a cylindrical sample with sufficiently fine graining and/or from the large sensitivity of the ${ }^{129} \mathrm{Xe}$ chemical shift.

From a more general point of view, these systematic numerical studies and their extensive confrontations with experiments for an extended range of parameters reveal that the usual Bessel solutions used to describe the echo modulations can be significantly erroneous due to finitesize and shape effects in the sample, or when non-uniform tipping is used. The importance of these last effects obviously questions the usual procedure which consists in resorting to an apparent dipolar field for taking diffusion effects into account $[57,58]$. This finding may have important consequences on the quantitative exploitation of spectra or images obtained with sequences based on $\mathrm{i}-\mathrm{MQC}$ in inhomogeneous media or magnetic fields.

\section{Acknowledgements}

This research program has been supported by ANR (ANR blanche DIPOL).

\section{References}

[1] G. Deville, M. Bernier, J. M. Delrieux, Phys. Rev. B 19 (1979) 5666-5688.

[2] D. Einzel, G. Eska, Y. Hirayoshi, T. Kopp, P. Wolfe, Phys. Rev. Lett. 53 (1984) 2312-2315.

[3] R. Bowtell, R. M. Bowley, P. Glover, J. Magn. Reson. 88 (1990) 643-651.

[4] H. Körber, E. Dormann, G. Eska, J. Magn. Reson. 93 (1991) 589-595.

[5] F. M. Alessandri, S. Capuani, B. Maraviglia, J. Magn. Reson. 156 (2002) 72-78.

[6] J. Jeener, J. Chem. Phys. 112 (2000) 5091-5094.

[7] A. Abragam, L. Goldman, Nuclear Magnetism: Order and Disorder, (Clarendon Press, Oxford 1982).

[8] J. Jeener, A. Vlassenbroek, P. Broekaert, J. Chem. Phys. 103 (1995) 1309-1332.

[9] J. Jeener, Encyclopedia of NMR, D. M. Grant, R. K. Harris (Eds.), 9, Wiley, 2002, pp. 642-679.

[10] H. Desvaux, Prog. NMR Spectrosc. in press. 10.1016/j.pnmrs.2012.11.001.

[11] W. S. Warren, S. Ahn, M. Mescher, M. Garwood, K. Ugurbil, W. Richter, R. R. Rizi, J. Hopkins, J. S. Leigh, Science 281 (1998) 247-251.

[12] W. S. Warren, W. Richter, A. H. Andreotti, B. T. Farmer, II, Science 262 (1993) 2005-2009. 
[13] S. Lee, W. Richter, S. Vathyam, W. S. Warren, J. Chem. Phys. 105 (1996) 874-900.

[14] Q. He, W. Richter, S. Vathyam, W. S. Warren, J. Chem. Phys. 98 (1993) 6779-6800.

[15] W. Richter, S. Lee, W. S. Warren, Q. He, Science 267 (1995) 654-657.

[16] S. Vathyam, S. Lee, W. S. Warren, Science 272 (1996) 92-96.

[17] Y. Huang, S. Cai, X. Chen, Z. Chen, J. Magn. Reson. 203 (2010) 100-107.

[18] S. Gutteridge, C. Ramanathan, R. Bowtell, Magn. Reson. Med. 47 (2002) 871-879.

[19] S. D. Kennedy, J. H. Zhong, Magn. Reson. Med. 52, (2004) 1-6.

[20] D. Z. Balla, G. Melkus, C. Faber, Magn. Reson. Med. 56 (2006) 745-753.

[21] G. Galiana, R. T. Branca, E. R. Jenista, W. S. Warren, Science 322 (2008) 421-424.

[22] V. Hoerr, A. Purea, C. Faber, Biophys. J. 99 (2010) 2336-2343.

[23] J. Jeener, Phys. Rev. Lett. 82 (1999) 1772-1775.

[24] J. Jeener, J. Chem. Phys. 116 (2002) 8439-8446.

[25] K. L. Sauer, F. Marion, P. J. Nacher, G. Tastevin, Phys. Rev. B 63 (2001) 184427.

[26] M. P. Ledbetter, I. M. Savukov, M. V. Romalis, Phys. Rev. Lett. 94 (2005) 060801.

[27] P. P. Zänker, J. Schmiedeskamp, H. W. Spiess, R. H. Acosta, Phys. Rev. Lett. 100 (2008) 213001.

[28] E. Baudin, M. E. Hayden, G. Tastevin, P.-J. Nacher, C. R. Chim. 11 (2008) 560-567.

[29] D. Candela, M. E. Hayden, P. J. Nacher, Phys. Rev. Lett. 73 (1994) 2587-2590.

[30] Y. Y. Lin, N. Lisitza, S. D. Ahn, W. S. Warren, Science 290 (2000) 118-121.

[31] P. J. Nacher, G. Tastevin, B. Villard, N. Piegay, F. Marion, K. L. Sauer, J. Low Temp. Phys. 121 (2000) 743-748.

[32] S. Y. Huang, Y. Y. Lin, N. Lisitza, W. S. Warren, J. Chem. Phys. 116 (2002) 10325-10337.

[33] S. Y. Huang, J. D. Walls, Y. Wang, W. S. Warren, Y. Y. Lin, J. Chem. Phys. 121 (2004) 6105-6109.

[34] M. E. Hayden, E. Baudin, G. Tastevin, P. J. Nacher, Phys. Rev. Lett. 99 (2007) 137602.

[35] D. J. Y. Marion, G. Huber, P. Berthault, H. Desvaux, ChemPhysChem 9 (2008) 1395-1401.

[36] D. J. Y. Marion, P. Berthault, H. Desvaux, Eur. Phys. J. D 51 (2009) 357-367.
[37] E. Jenista, R. Branca, W. Warren, J. Magn. Reson. 196 (2009) 74-77.

[38] M. Mishkovsky, U. Eliav, G. Navon, L. Frydman, J. Magn. Reson. 200 (2009) 142-146.

[39] X. Tang, H. Ong, K. Shannon, W. S. Warren, Magn. Reson. Imaging 21 (2003) 1141-1149.

[40] J. D. Walls, F. K. H. Phoa, Y. Y. Lin, Phys. Rev. B 70 (2004) 174410.

[41] A. Abragam, M. Chapellier, J. F. Jacquinot, M. Goldman, J. Magn. Reson. 10 (1973) 322-346.

[42] Y. M. Chen, R. T. Branca, W. S. Warren, J. Chem . Phys. 136, (2012) 204509.

[43] J. Ardenkjaer-Larsen, B. Fridlund, A. Gram, G. Hansson, L. Hansson, M. Lerche, R. Servin, M. Thaning, K. Golman, Proc. Natl. Acad. Sci. U.S.A. 100 (2003) 10158-10163.

[44] M.E. Merritt, C. Harrison, W. Mander, C.R. Malloy, A.D. Sherry, J. Magn. Reson. 189 (2007) 280-285.

[45] H. Desvaux, P. Berthault, Prog. Nucl. Magn. Reson. Spectrosc. 35 (1999) 295-340.

[46] M. Goldman, H. Desvaux, Chem. Phys. Lett. 256 (1996) 497-501.

[47] J. G. Powles, P. Mansfield, Phys. Lett. 2 (1962) 58 59.

[48] H. Desvaux, T. Gautier, G. Le Goff, M. Pétro, P. Berthault, Eur. Phys. J. D 12 (2000) 289-296.

[49] P. Berthault, G. Huber, H. Desvaux, Prog. Nucl. Magn. Reson. Spectrosc. 55 (2009) 35-60.

[50] H. L. Clever, IUPAC Solubility Data Series, (Pergamon Press, 1979).

[51] D. J. Y. Marion, G. Huber, L. Dubois, P. Berthault, H. Desvaux, J. Magn. Reson. 187 (2007) 78-87.

[52] D. J. Y. Marion, H. Desvaux, J. Magn. Reson. 193 (2008) 153-157.

[53] E. Baudin, K. Safiullin, S. W. Morgan, P. J. Nacher, J. Phys.: Conf. Ser. 294 (2011) 012009.

[54] T. Enss, S. Ahn, W. S. Warren, Chem. Phys. Lett. 305 (1999) 101-108.

[55] C. B. Cai, Z. Chen, S. H. Cai, J. H. Zhong J. Magn. Reson. 172, (2005) 242-253.

[56] H. T. Edzes, J. Magn. Reson. 86 (1990) 293-303.

[57] I. Ardelean, E. Kossel, R. Kimmich, J. Chem. Phys. 114 (2001) 8520-8529.

[58] C. Ramanathan, R. Bowtell, J. Chem. Phys. 114 (2001) 10854-10859. 